\title{
Análisis y evaluación de la enfermera referente del paciente en hemodiálisis
}

\author{
Loreto Sáez Rodríguez - Ma Jesús Pérez Paz - Cristina Valiño Pazos - Silvia Castro Fernández
}

Unidad de Diálisis. Complexo Hospitalario Universitario de A Coruña

\section{Resumen}

La enfermera referente es una pieza clave para ofertar cuidados de calidad. Nuestro objetivo ha sido conocer como evalúan los pacientes la figura de la enfermera referente en nuestra unidad de hemodiálisis, para revisar y actualizar su rol y sus tareas. Se seleccionó una muestra de 51 pacientes crónicos que acuden a hemodiálisis en nuestro centro a los que se entregó una encuesta anónima, autoadministrada, diseñada y validada por el personal de la unidad.

Los resultados obtenidos indican que los pacientes evalúan positivamente a la enfermera referente confirmándose la utilidad y relevancia de la misma. Sus respuestas definen un perfil básico de enfermera referente: una profesional a la que conocen por su nombre, amable y cortés, con la que se comunican frecuentemente, con extensa formación y experta en su trabajo, capacitada y dispuesta a resolver las dudas y problemas que los pacientes plantean. En definitiva, su enfermera de confianza. Las aportaciones de los encuestados para mejorar los cuidados, nos sugieren proporcionar más información sobre la dieta y proporcionar espacios de intimidad para la comunicación enfermera-paciente.

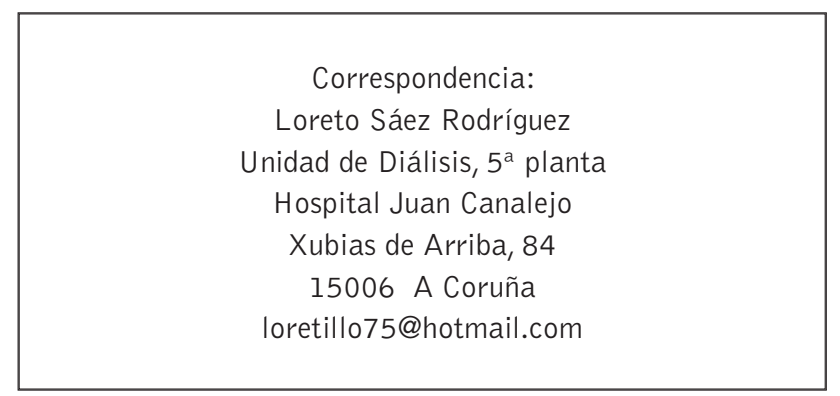

PALABRAS CLAVE:

- ENFERMERA REFERENTE

- HEMODIÁLISIS

- SATISFACCIÓN PACIENTE

- CALIDAD CUIDADOS

\section{Analysis and assessment of the referring nurse of patients on haemodialysis}

\section{Abstract}

The referring nurse is a key element in offering quality care. Our aim was to find out how patients assess the referring nurse in our haemodialysis unit, in order to review and update her role and tasks. A sample of 51 chronic patients undergoing haemodialysis in our centre was selected and these patients were given an anonymous self-administered questionnaire, designed and validated by staff of the unit.

The results obtained show that patients value the referring nurse positively, confirming her usefulness and relevance. Their replies define a basic profile for a referring nurse: a professional they know by name, friendly and polite, with whom they communicate frequently, with extensive training and an expert in her work, capable of and willing to overcome the doubts and problems that patients raise. In short, the nurse they trust. The contributions of the questionnaire respondents for improving care suggest that we provide more information about diet and provide areas of privacy for nurse-patient communication. 


\section{KEY WORDS:}

- REFERRING NURSE

- HAEMODIALYSIS

PATIENT SATISFACTION

- CARE QUALITY

\section{Introducción}

La enfermera referente ${ }^{1}$ nace en nuestra unidad hace más de quince años. Desde entonces hemos desarrollado, a través de ella y como apoyo al paciente crónico, funciones de tratamiento, educación sanitaria, soporte psicológico o rehabilitación terapéutica. Sabemos que nuestra unidad no es pionera en la implantación y desarrollo de esta figura, pues otras áreas como psiquiatría, medulares o atención primaria también cuentan con la labor de enfermeras referentes para optimizar sus recursos.

En nuestra unidad, sus funciones y tareas están descritas en un protocolo específico, que se revisa anualmente; a cada una de las enfermeras de hemodiálisis se le asignan tres pacientes crónicos de forma aleatoria y con carácter permanente, a los que tutela y gestiona ${ }^{2}$ desde su llegada para iniciar tratamiento, orientándolos en su nuevo problema de salud y formándolos en todo lo relativo al tratamiento renal sustitutivo (dieta, medicación, ejercicio, estilo de vida...). También es responsable del buen estado y actualización de la historia clínica, revisándola mensualmente y realizando un resumen semestral de la misma.

Hace seis años, se instauró la entrevista individual y personalizada, que fomenta la educación programada y a demanda, garantizando unos cuidados enfermeros de calidad e intentando promover el nivel máximo de independencia en el autocuidado.

Este tipo de organización de los cuidados enferme$\operatorname{ros}^{3}$, en los que una enfermera actúa como responsable y referencia única para el paciente, presenta una serie de beneficios: aumenta la responsabilidad de la enfermera designada, con el correspondiente aumento de la calidad de cuidados. Mejora la continuidad de los cuidados enfermeros, facilitando el intercambio con otros profesionales. La enfermera da a conocer su trabajo al paciente y a su familia ${ }^{4}$, repercutiendo favorablemente en la sociedad, en cuanto a reconocimiento profesional se refiere. La satisfacción personal se encuentra aumentada.

\section{Objetivos}

- Evaluar el grado de satisfacción del paciente crónico en relación a la figura de la enfermera de referencia.

- Describir la utilidad y relevancia de la enfermera referente.

- Conocer el grado de coincidencia entre funciones teóricas y funciones reales desarrolladas.

- Valorar la incorporación de nuevas tareas y roles, sugeridos o demandados por nuestros pacientes a través de la encuesta de satisfacción.

\section{Material y Método}

Tras una recopilación bibliográfica sobre el tema se revisó el protocolo de actuación de la enfermera de referencia vigente en nuestra unidad. Se incluyó en el estudio a todos los pacientes crónicos en tratamiento sustitutivo exceptuando a los pacientes que habían comenzado su tratamiento en el último mes ( 51 individuos de un total de 55).

Para evaluar la figura de la enfermera referente, diseñamos una encuesta autoadministrada, con respuestas predefinidas tipo ítem (figural), modificando un cuestionario de Mira $\mathrm{JJ}^{5}$ sobre calidad percibida de la asistencia hospitalaria, y la validamos a través de grupo discusión de expertos. Dicha encuesta se entregó personalmente a todos los pacientes de nuestra unidad de crónicos acompañada de una carta de presentación, en la que se detallaban los objetivos del estudio, el modo de cumplimentación y la posibilidad de solicitar ayuda si se precisara, tanto al personal de la unidad, como a sus familiares 0 allegados. Para garantizar el anonimato de los encuestados, las encuestas se recogieron a través de un buzón situado en los vestuarios de la sala de hemodiálisis, en el que cada paciente pudo depositar su test en sobre cerrado. 


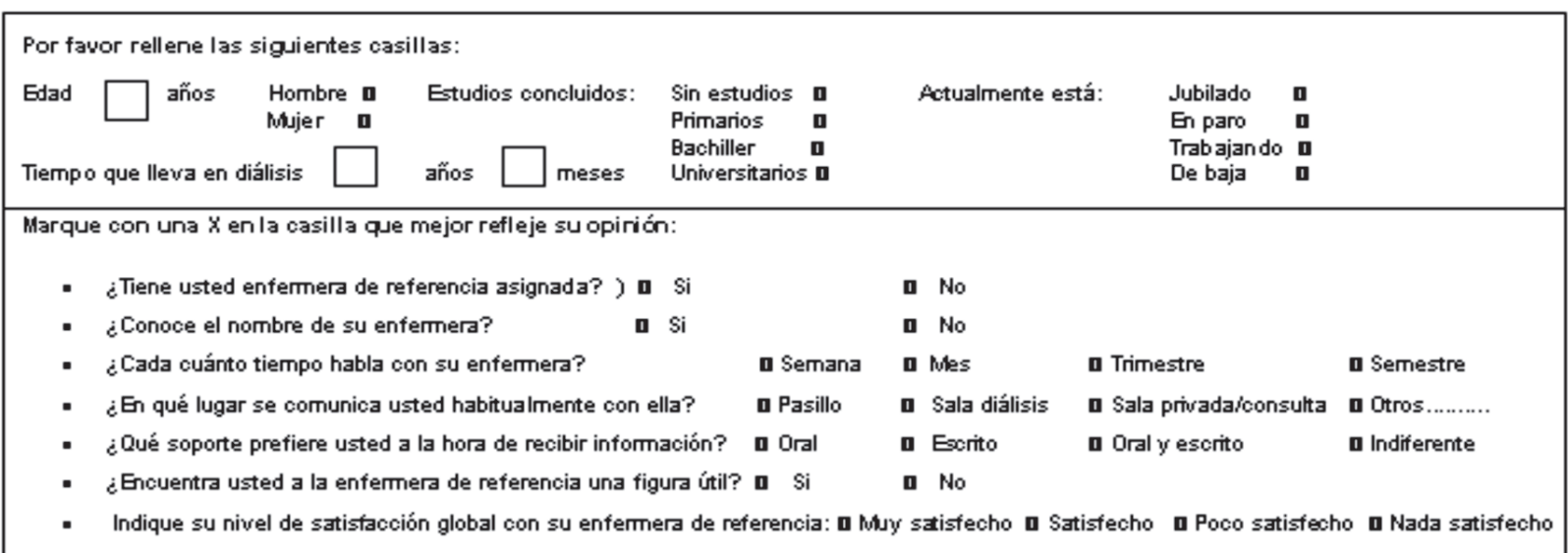

Señale con una cruz su opirión acerca de su enfermera de referencia

MI ENFERMERA DE REFERENCIA:

\begin{tabular}{|c|c|c|c|c|}
\hline & $\begin{array}{l}\text { Completamente } \\
\text { de acuerdo }\end{array}$ & De acuerdo & En desacuerdo & $\begin{array}{l}\text { Completamente } \\
\text { en desacuerdo }\end{array}$ \\
\hline \multicolumn{5}{|l|}{ Tiene la preparación adecuada para realizar su trabajo } \\
\hline \multicolumn{5}{|l|}{ Me dedica el tiempo necesario } \\
\hline \multicolumn{5}{|l|}{ Cumple lo que dice que va a hacer } \\
\hline \multicolumn{5}{|l|}{ Muestra interés en solucionar mis problemas } \\
\hline \multicolumn{5}{|l|}{ Comprende mis necesidades } \\
\hline \multicolumn{5}{|l|}{ Me transmite confianza y me aporta seguridad } \\
\hline \multicolumn{5}{|l|}{ Me trata con amabilidad y cortesía } \\
\hline \multicolumn{5}{|l|}{ Me informa sobre mi problema de salud } \\
\hline \multicolumn{5}{|l|}{ Hace un seguimiento de mi dieta } \\
\hline \multicolumn{5}{|c|}{ Revisa mi tratamiento y resuelve mis dudas de medicación } \\
\hline Si es necesario se pone en contacto con mi familia & & & & \\
\hline
\end{tabular}

ENCUESTA (Figura 1)

Figura 1. Encuesta

Periodo de estudio: establecimos como fecha de inicio de entrega de los cuestionarios el 8-II-2008 y como fecha límite de recogida el 15-III-2008, tras haber decidido respetar un plazo de cumplimentación de un mes.

Tras el análisis de los datos obtenidos se procedió a la implementación de las posibles medidas correctoras. La tabulación y análisis de los dato se hizo a través del programa informático SPSS 15.00.

\section{Resultados}

De las 51 encuestas entregadas, fueron cumplimentadas de modo correcto para su estudio 33. Los resultados referentes a las variables socioculturales que hemos estudiado y que definen la muestra son los siguientes: media de edad, distribución por sexos y tiempo que los encuestados Ilevan con tratamiento de hemodiálisis, nivel de estudios y situación laboral actual se recogen en las figuras 2,3 y 4 . En cuanto a las variables relativas a preguntas específicas, obtuvimos los siguientes resultados:

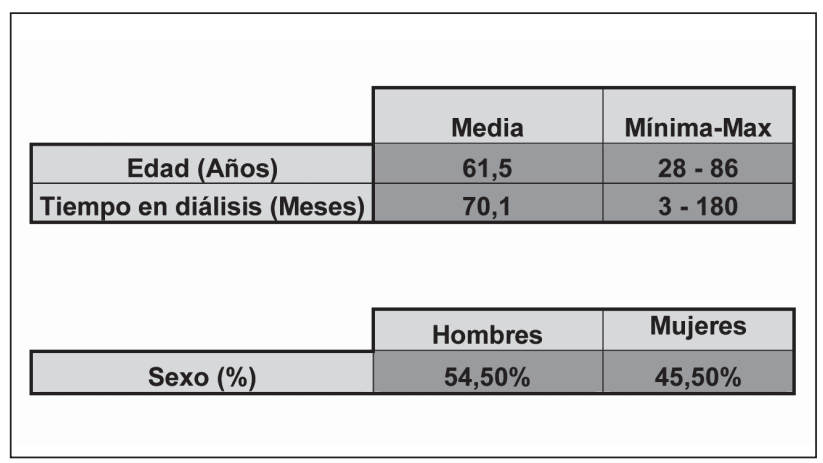

Figura 2. Distribución por sexo y edad 


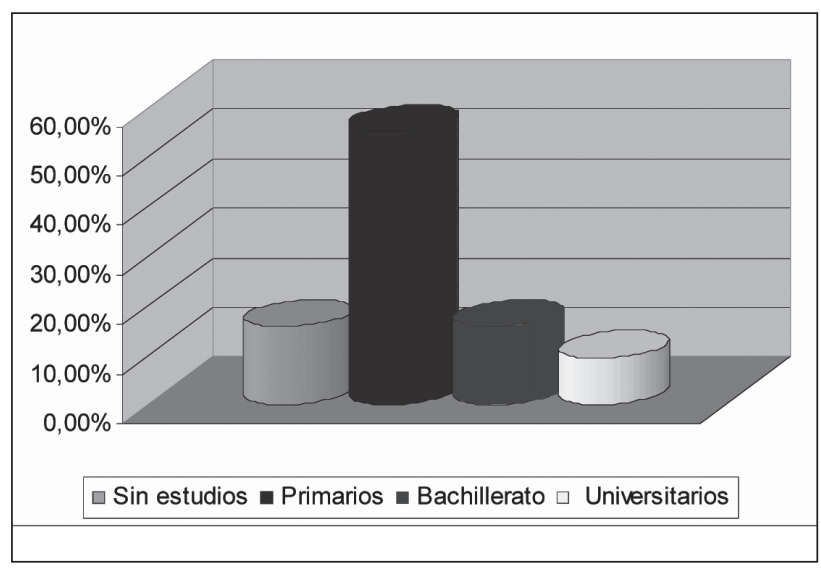

Figura 3. Nivel de estudios

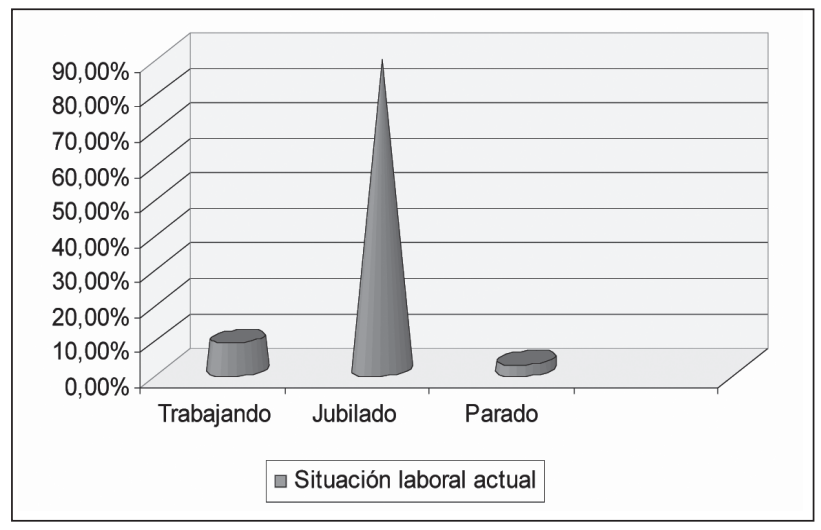

Figura 4. Situación laboral actual
Cuando se valoró el grado de utilidad de la enfermera de referencia para nuestros usuarios comprobamos que la consideran una figura útil un $93.3 \%$. Asimismo, el nivel de satisfacción también fue valorado muy positivamente, de modo que un $51.6 \%$ de nuestros pacientes se define como "satisfecho" y un $45.2 \%$ se considera "muy satisfecho" con la enfermera que tiene asignada.

En el apartado de "opinión acerca de su enfermera de referencia" que fue valorado puntuando una escala tipo Likert que cuantificaba el grado de adherencia personal, y donde las posibilidades de elección abarcaban cuatro opciones, variando de "completamente de acuerdo" a " completamente en desacuerdo", podemos observar que, para todas las cuestiones, la mayoría ha afirmado estar "completamente de acuerdo" y/o "de acuerdo", con la única excepción del enunciado que valora la información sobre la dieta, en la que por un porcentaje ligeramente superior, la mayoría se muestra simplemente "de acuerdo" (figura 5).

En cuanto a la incorporación de nuevas tareas y roles, obtenemos una demanda de mayor educación sanitaria referida a la dieta, solicitudes de información escrita y un lugar privado para una comunicación más íntima y confortable.

Respondieron afirmativamente un $97 \%$ al ser preguntados si tenían enfermera de referencia asignada y la misma proporción confirmó conocer su nombre. En cuanto a la frecuencia de comunicación/encuentros personales con su enfermera referente un $63 \%$ lo hace semanalmente, mensualmente un $29.5 \%$ y cada trimestre o semestre un $4 \%$. El lugar de encuentro es casi siempre $(93.1 \%$ ) la sala de diálisis, frente a otros lugares menos habituales, como el pasillo o una consulta privada (en nuestra unidad, la primera entrevista personal siempre se desarrolla en privado, a través de una cita concertada).

En relación al soporte informativo, la mitad de nuestros usuarios señala que prefiere recibir la información oral, una décima parte escrita y a un $34.5 \%$ le gustaría recibirla de forma combinada, es decir, oral y escrita.

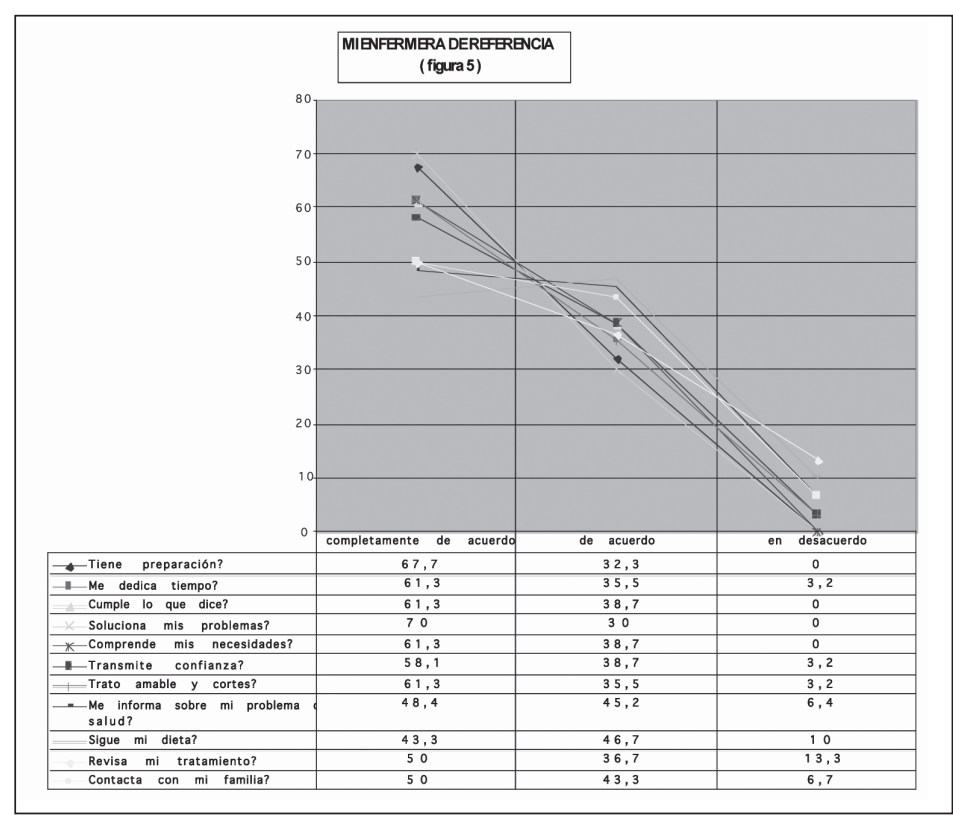

Figura 5. Opinión acerca de su enfermera de referencia 


\section{Discusión}

Cuando nos planteamos llevar a cabo este estudio, uno de nuestros objetivos fue la descripción de la utilidad y relevancia de la figura de la enfermera de referencia en nuestra unidad. Los resultados obtenidos, en concordancia con los de otros autores ${ }^{6}$, dan respuesta a los dos primeros objetivos planteados en el estudio, puesto que confirman un nivel alto de satisfacción en nuestros clientes que perciben la utilidad de la enfermera referente.

La comparación de la figura de la enfermera referente diseñada teóricamente con los resultados obtenidos en el apartado "opinión acerca de su enfermera de referencia", y la valoración de las respuestas para conocer si las funciones desempeñadas por la enfermera referente coinciden con las expectativas o demandas de nuestros pacientes permite estudiar la concordancia entre las tareas definidas por el protocolo en nuestra unidad y las reconocidas por nuestros usuarios, obteniendo una evaluación favorable: las funciones teóricas coinciden con las actividades que el paciente percibe. La mayoría de nuestros pacientes consideran la enfermera de referencia como una figura útil de la que se encuentran satisfechos. Sus respuestas nos indican que todos ellos son capaces de reconocer cual de nosotras es "su enfermera", que la comunicación y transmisión de la información periódica está asegurada y es llevada a cabo de forma habitual en la sala de hemodiálisis y nos permiten comprobar que la gran mayoría afirman sentirse completamente satisfechos con sus actuaciones.

Nuevos retos se nos plantean tras la reflexión sobre los resultados del estudio: admitimos que nos produce una grata sensación comprobar que nuestra labor es bien valorada y reconocida por aquellos a quienes van dirigidos nuestros cuidados pero, sin embargo, aceptamos que hay ciertos aspectos a tener en cuenta para mejorar, como revisar la forma y contenido de la información relativa a la dieta o asegurarnos de que la comunicación pueda tener lugar en un ambiente privado.

También para adaptarnos a las nuevas metodologías de trabajo enfermero, debemos ir incorporando a nuestra práctica diaria herramientas de mejora como son el uso de lenguajes estandarizados para el registro y valoración. En la actualidad, estamos introdu- ciendo en la práctica diaria un nuevo instrumento de trabajo: "La guía de valoración por indicadores del paciente con IRCT en hemodiálisis", diseñada este mismo año por el personal de la unidad y orientada a implantar la terminología NANDA-NIC-NOC en nuestros cuidados lo que permitirá a la enfermera referente hacer una evaluación basada en un lenguaje común para lograr un registro unificado de los diagnósticos enfermeros relativos a cada paciente. En un futuro, ligaremos cada diagnóstico a sus correspondientes intervenciones para completar la valoración con la definición de tareas en cada caso.

En definitiva, trataremos de aportar todo cuanto nos permita aumentar la calidad y eficacia de nuestros cuidados para seguir siendo referente y apoyo de nuestros pacientes

\section{Bibliografía}

1. Martínez Riera JR. Consulta de enfermería a demanda en Atención Primaria. Reflexión de una necesidad. Revista de Administración Sanitaria Siglo XXI. 2003; 1(3):425-440.

2. Andrade García F, Expósito Carbal C, García Palacios R. Caso clínico: un paciente etiquetado de difícil. Rev Soc Esp Enferm Nefrol. 2005; 8 (3):6769.

3. Aquino Oliveira M. Experiencia en enfermera tutora. http://www.lesionadomedular.com/cg-bin/articulos/cat_articulos.cgi?pag=20\&id_categoria=1 $1 \&$ area $=1$ (consultado el 13/12/07)

4. Torres Pérez L, Morales Asencio JM. Participación familiar en el cuidado del paciente crítico. Tempos Vitales Revista Internacional para el Cuidado del Paciente Crítico. 2004; 4 (1):18-25.

5. Mira JJ, Aranaz J, Rodríguez-Marín J, Buil JA, Castell M, Vialler J. SERVQHOS: un cuestionario para evaluar la calidad percibida de la asistencia hospitalaria. Medicina Preventiva.1998; 4:8-12.

6. Sasot JA. La enfermera de referencia, su satisfacción laboral y la del paciente. Enfer Clin. 1998; 8 (2): 31-36. 